\title{
High-Resolution Crystal Structure of Chloroplastic Ribose-5-Phosphate Isomerase from Chlamydomonas reinhardtii-An Enzyme Involved in the Photosynthetic Calvin-Benson Cycle
}

\author{
Théo Le Moigne ${ }^{1,2} \mathbb{D}$, Pierre Crozet ${ }^{1,3}$, Stéphane D. Lemaire ${ }^{1,4}$ and Julien Henri $1, * \mathbb{C}$ \\ 1 Laboratoire de Biologie Moléculaire et Cellulaire des Eucaryotes, UMR8226, Institut de Biologie \\ Physico-Chimique, Sorbonne Université, CNRS, 75005 Paris, France; lemoigne@ibpc.fr (T.L.M.); \\ pierre.crozet@sorbonne-universite.fr (P.C.); lemaire@ibpc.fr (S.D.L.) \\ 2 Faculty of Sciences, Doctoral School of Plant Sciences, Université Paris-Saclay, 91190 Saint-Aubin, France \\ 3 Polytech Sorbonne, Sorbonne Université, 4 place Jussieu, 75005 Paris, France \\ 4 Laboratoire de Biologie Computationnelle et Quantitative, UMR7238, Institut de Biologie Paris-Seine, \\ Sorbonne Université, CNRS, 4 place Jussieu, 75005 Paris, France \\ * Correspondence: julien.henri@sorbonne-universite.fr
}

Received: 31 August 2020; Accepted: 19 October 2020; Published: 21 October 2020

\begin{abstract}
The Calvin-Benson cycle is the key metabolic pathway of photosynthesis responsible for carbon fixation and relies on eleven conserved enzymes. Ribose-5-phosphate isomerase (RPI) isomerizes ribose-5-phosphate into ribulose-5-phosphate and contributes to the regeneration of the Rubisco substrate. Plant RPI is the target of diverse post-translational modifications including phosphorylation and thiol-based modifications to presumably adjust its activity to the photosynthetic electron flow. Here, we describe the first experimental structure of a photosynthetic RPI at $1.4 \AA$ resolution. Our structure confirms the composition of the catalytic pocket of the enzyme. We describe the homo-dimeric state of the protein that we observed in the crystal and in solution. We also map the positions of previously reported post-translational modifications and propose mechanisms by which they may impact the catalytic parameters. The structural data will inform the biochemical modeling of photosynthesis.
\end{abstract}

Keywords: photosynthesis; chloroplast; Calvin-Benson cycle; protein structure; X-ray crystallography; ribose isomerase; post-translational modification; redox; oligomerization; protein complexes

\section{Introduction}

Photosynthesis is the biological process allowing the conversion of light energy into chemical energy through fixation of atmospheric carbon [1]. Fixation of carbon dioxide occurs through the Calvin-Benson cycle (CBC) by carboxylation of the acceptor pentose ribulose-1,5-bisphosphate (RubP) by Rubisco [2]. RubP is generated by phosphorylation of ribulose-5-phosphate (Ru5P) by phosphoribulokinase (PRK). Prior to this phosphorylation step, ribose-5-phosphate (R5P) carbonyl group is transferred from carbon 1 to carbon 2 . This reaction is catalyzed by the metabolic enzyme ribose-5-phosphate isomerase (RPI) [3]. Two forms of RPI have been classified: type $A\left(R^{2} I_{A}\right)$ and type $\mathrm{B}\left(\mathrm{RPI}_{\mathrm{B}}\right) . \mathrm{RPI}_{\mathrm{A}}$ is ubiquitous in all species while $\mathrm{RPI}_{\mathrm{B}}$ is only present in some bacteria and protozoans [4]. Beside its critical role in the $\mathrm{CBC}, \mathrm{RPI}_{\mathrm{A}}$ is implicated in the non-oxidative branch of the pentose phosphate pathway (PPP) [5]. $\mathrm{RPI}_{\mathrm{A}}$ function in the PPP makes it an attractive target for drug development in the treatment of trypanosomatid-caused diseases [4]. $\mathrm{RPI}_{\mathrm{A}}$ structures and catalytic 
mechanism (EC 5.3.1.6) have been described in several organisms, but the structure of photosynthetic chloroplast localized $\mathrm{RPI}_{\mathrm{A}}$ has never been reported to date. Analysis of RPI enzymatic kinetics has been principally performed on $\mathrm{RPI}_{\mathrm{B}}$ in several species, and research on $\mathrm{RPI}_{\mathrm{A}}$ enzymology is comparatively scarcer. The catalytic mechanism of $\mathrm{RPI}_{\mathrm{A}}$ has nonetheless been established in several species and summarized in a recent review [4].

Chlamydomonas reinhardtii is a model unicellular photosynthetic green alga [6]. Its nuclear genome encodes two RPI at loci Cre03.g187450.t1.2 and Cre07.g314600.t1.2 [7], the protein products of which are respectively named RPI1 and RPI2. Both proteins are predicted to be addressed to the chloroplast according to the Predalgo tool [8] and in agreement with the chloroplast localization of the CBC and PPP in C. reinhardtii $[9,10]$. Among RPI1 and RPI2, RPI1 was selected as the most probable contributor to the CBC because its transcripts are 16 to 199 times more abundant than that of RPI2 in C. reinhardtii [11] and because accumulation of RPI1 transcript varies according to a night/day cycle, mirroring luminous flux.

In C. reinhardtii, the estimated CrRPI1 protein quantity is 0.34 amol per cell and $2.5 \mu \mathrm{mol}^{-L^{-1}}$ in the chloroplast [12]. Considering the cellular concentration of metabolites [12,13], the approximate molar ratio of substrate or product to CrRPI 1 is respectively estimated at 80 and 50 . In order to adapt to the variations of metabolites concentration in the chloroplast stroma, CBC enzymatic activities are generally regulated according to the environmental light $[14,15]$. These regulations occur through several signaling pathways, e.g., dithiol-disulfide exchanges, S-thiolations, S-glutathionylations, and S-nitrosylations [15-17]. The stroma magnesium concentration is also known to influence the catalytic activity of CBC enzymes $[18,19]$. Post-translational redox modifications of RPI were detected from C. reinhardtii proteomic studies [20]. Phosphorylated peptides of CBC enzymes were also identified from C. reinhardtii extracts [21], suggesting a catalytic post-translational regulation by reversible phosphorylation.

Here, we present the first experimental structure of a chloroplastic RPI. We describe the structural characteristics that classifies CrRPI1 in the $\mathrm{RPI}_{\mathrm{A}}$ family. We map the positions of previously reported post-translational modifications and propose mechanisms by which they may impact the catalytic parameters.

\section{Results}

\subsection{CrRPI1 Structural Folds}

We solved the crystal structure of CrRPI1 in the P2 ${ }_{1}$ space group at $1.40 \AA$ resolution (Table 1). Model building of the two subunits of the asymmetric unit achieved the placement of 235 amino acid residues in the first subunit and 237 in the second (Figure 1A). The residues of each subunit were numbered according to uniProtKB entry A8IRQ1. The high resolution of the dataset further allowed us to position eight sulfates, two sodiums, and 511 water molecules in the crystal asymmetric unit. 
Table 1. Crystallographic data collection and refinement statistics.

\begin{tabular}{|c|c|}
\hline & CrRPI1 \\
\hline Wavelength $(\AA)$ & 0.979 \\
\hline Resolution range $(\AA)$ & $40.22-1.40(1.45-1.40)$ \\
\hline Space group & $\mathrm{P} 2_{1}$ \\
\hline Unit cell & 45.1563 .9580 .919096 .1390 \\
\hline Total reflections & $613,155(55,349)$ \\
\hline Unique reflections & $89,012(8611)$ \\
\hline Multiplicity & $6.9(6.4)$ \\
\hline Completeness (\%) & $99.6(96.5)$ \\
\hline Mean I/sigma(I) & $20.58(1.24)$ \\
\hline Wilson B-factor $\left(\AA^{2}\right)$ & 21.45 \\
\hline R-merge & $0.0519(1.272)$ \\
\hline R-meas & $0.05611(1.382)$ \\
\hline R-pim & $0.02114(0.5327)$ \\
\hline $\mathrm{CC} 1 / 2$ & $0.999(0.382)$ \\
\hline $\mathrm{CC}^{*}$ & $1(0.744)$ \\
\hline Reflections used in refinement & $88,983(8601)$ \\
\hline Reflections used for R-free & 2000 (194) \\
\hline R-work & $0.1594(0.3457)$ \\
\hline$R$-free & $0.1774(0.3813)$ \\
\hline CC (work) & $0.972(0.730)$ \\
\hline $\mathrm{CC}($ free $)$ & $0.967(0.836)$ \\
\hline $\begin{array}{l}\text { Number of non-hydrogen } \\
\text { atoms }\end{array}$ & 4206 \\
\hline macromolecules & 3648 \\
\hline ligands & 47 \\
\hline solvent & 511 \\
\hline Protein residues & 474 \\
\hline RMS (bonds) (Å) & 0.011 \\
\hline RMS (angles) (deg) & 1.18 \\
\hline Ramachandran favored (\%) & 99.79 \\
\hline Ramachandran allowed (\%) & 0.21 \\
\hline Ramachandran outliers (\%) & 0.00 \\
\hline Rotamer outliers (\%) & 1.00 \\
\hline Clashscore & 2.69 \\
\hline Average B-factor $\left(\AA^{2}\right)$ & 25.46 \\
\hline macromolecules & 23.78 \\
\hline ligands & 47.04 \\
\hline Solvent & 35.49 \\
\hline
\end{tabular}

Statistics for the highest-resolution shell are shown in parentheses.

A

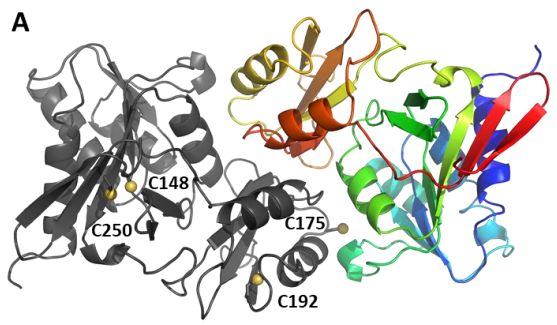

B

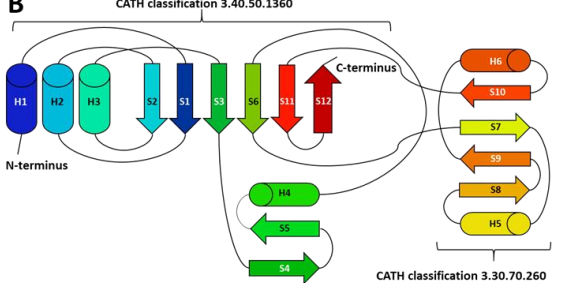

C
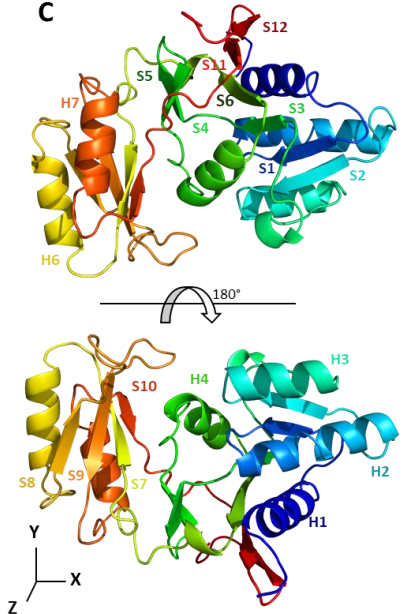

Figure 1. Crystal structure of CrRPI1. (A) Cartoon representation of the CrRPI1 crystallographic dimer colored in grey for the first subunit and from blue (N-terminus) to red (C-terminus) for the second subunit. (B) Two-dimensional representation of secondary structures of CrRPI1 colored as the second subunit in (A). (C) Cartoon representation of a CrRPI1 monomer. Two views of the protein are represented as in (A) and rotated by $180^{\circ}$ on the $x$-axis. Secondary structures are annotated as in (A). 
Each monomer is composed of six alpha helices and twelve beta strands distributed into two domains. Domain 1 spans residues 29 to 160 and 246 to 264, folds according to the Rossmann topology, and is part of an unnamed structural superfamily (CATH classification 3.40.50.1360). Domain 2 is inserted in between the two parts of domain 1, spans residues 161 to 245, and is classified as an ACT domain (CATH classification 3.30.70.260) (Figure 1B).

Our model aligns with 62 entries of the Protein data bank with an average RMSD lower than $1.8 \AA$. The closest similarity is with the Ribose 5-phosphate isomerase from Plasmodium falciparum (PDB $2 \mathrm{~F} 8 \mathrm{M}, \mathrm{RMSD}=0.852 \AA$ over 229 aligned $\mathrm{C} \alpha)$. CrRPI1 secondary structure content is distributed as follows: $\alpha$ helix h1 (residues 33-45), $\beta$ strand s1 (52-56), h2 (60-75), s2 (81-85), h3 (88-97), s3 (112-115), s4 (118-121), s5 (124-127), h4 (135-143), s6 (146-152), s7 (167-171), h5 (176-185), s8 (193-196), s9 (211-216), h6 (225-233), s10 (238-240), s11 (249-254) and s12 (257-262) (Figure 1C). In addition, residues 104, 105, 106 and residues 152, 153, 154 form two $3_{10}$ helices. Residues 22 to 28 and 265 to 269 at amino- and carboxy-termini were not modelled because of a lack of interpretable electron density. The secondary structure content and organization is the same as a canonical RPI previously reported (PfRPI, PDB 2F8M, [22]) except for the presence of CrRPI1 310 helix formed by residues 104-106 that is absent from PfRPI.

Domain 1 is composed of helices 1,2,3, and 4, with strands 4 and 5 forming a small antiparallel sheet and strands $1,2,3,6$, and 12 that arrange in a parallel sheet completed by strand 11 in antiparallel orientation. These secondary structures are organized in a three-layer sandwich composed of helices 1, 2, 3 for the first layer, the parallel sheet in the second layer and by strand 4, 5, helix 4 and the two $3_{10}$ helices for the third layer. Domain 2 is relatively smaller with helices 5 and 6 forming the first stratum of a two-layer sandwich and strands 7, 8, 9, and 10 organized as an antiparallel sheet forming the second layer. The first domain contains the catalytic pocket, while the second was proposed to play a role in the oligomerization of $\mathrm{RPI}_{\mathrm{A}}[4]$.

\subsection{CrRPI1 is a Homodimer}

$\mathrm{RPI}_{\mathrm{A}}$ has been described as a functional dimer in solution $[3,23,24]$ in bacteria and protozoa or as a functional tetramer in species from archaea and fungi taxa [4]. However, nothing was described for Viridiplantae enzymes. In our study, CrRPI1 elutes with the apparent molecular weight of a homodimer in solution over two different gel filtration matrices (Figure 2A). Small angle X-rays scattering (SAXS) curves obtained after size-exclusion chromatography of the purified protein (SEC-SAXS, Figure 2B) compute a molecular weight between $49.25 \mathrm{kDa}$ and $56.2 \mathrm{kDa}$, fitting with two monomers of $27.55 \mathrm{kDa}$ each. The homodimeric state is also observed in the crystal asymmetric unit (Figure 1A) with $\alpha$ helices $\mathrm{h} 4, \mathrm{~h} 5$ and $\mathrm{h} 6$ and $\beta$ strands s10 contributing to the interface. CrRPI1 dimer was aligned with Plasmodium falciparum RPI dimer (PDB entry 2F8M) with an RMSD $=2.115 \AA$ Aver 438 equivalent atoms. Both dimers have the same quaternary organization, with corresponding subunits positioned in virtually identical positions. The only significant difference lies in the $3_{10}$ helix formed by residues 104-106 of CrRPI1 that is structured as a loop in PfRPI. The interface between two subunits of CrRPI1 is calculated from the arrangement of the two subunits forming the asymmetric unit (Figure 3A) by the PDBe-PISA server v1.52 [25]. The subunit A-to-subunit B interface is $1165 \AA^{2}$ wide and has a computed $\Delta^{\mathrm{i}} \mathrm{G}=-19.3 \mathrm{kcal} / \mathrm{mol}(p$-value $=0.027)$. In comparison, PfRPI dimer interface is more extended with a surface of $1340.8 \AA^{2}$ and an equivalent computed $\Delta^{\mathrm{i}} \mathrm{G}=-19.4 \mathrm{kcal} / \mathrm{mol}(p$-value $=0.048)$. CrRPI1 interface is composed by the 33 following residues: P85, T103, L104, D105, P108, K109, L135, R136, K138, M139, V140, M142, A143, V172, Q173, F174, C175, H176, K177, Y178, T179, R82, D207, N208, S209, N210, L232, G233, D235, G236, V237, V238, D239. It is stabilized by three hydrogen bonds between residues $\mathrm{Y} 178_{\mathrm{A}}\left(\alpha\right.$ helix h5) and D105 ${ }_{\mathrm{B}}\left(3_{10}\right.$ helix 104-106), F174 $4_{\mathrm{A}}$ and $\mathrm{N} 208_{\mathrm{B}}$, D105 ${ }_{\mathrm{A}}$ and $\mathrm{Y} 178_{\mathrm{B}}$, and one salt bridge between $\mathrm{K} 177_{\mathrm{A}}$ and D105 ${ }_{\mathrm{B}}$ (Figure $3 \mathrm{C}$ ). PfRPI possesses similar pairs $\mathrm{Y} 145_{\mathrm{A}}-\mathrm{E} 74_{\mathrm{B}}, \mathrm{N} 177_{\mathrm{A}}-\mathrm{F} 143_{\mathrm{B}}$, and $\mathrm{E} 74_{\mathrm{A}}-\mathrm{K} 146_{\mathrm{B}}$ (main chain carbonyl) in bonding distance, but no salt bridge and additional hydrogen bonds that are absent in CrRPI1 homodimer. While CrRPI1 
dimerization interface residues are strictly conserved in the algae taxon, they are relatively poorly conserved throughout evolution (Figure 3B and Figure S1) [26].

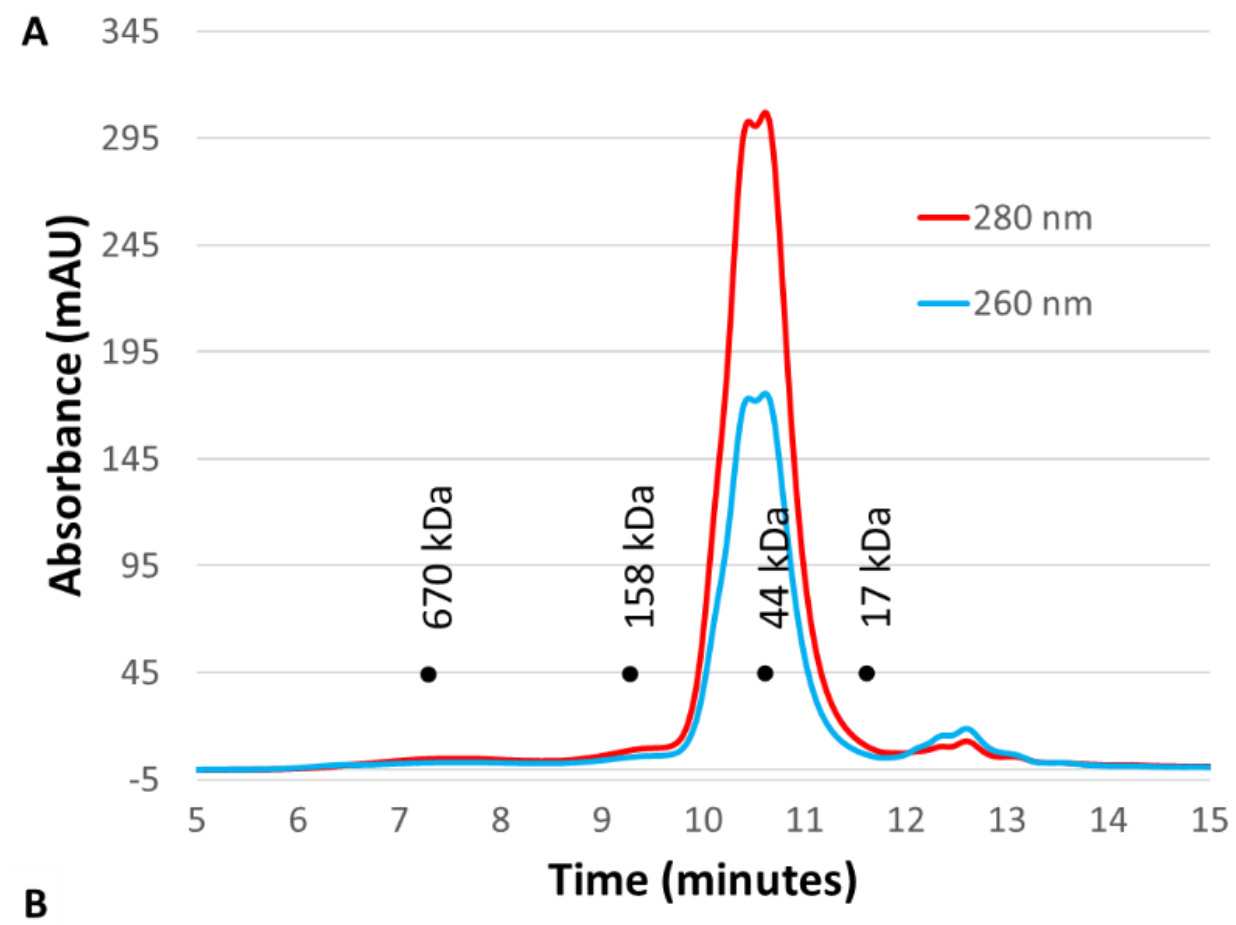

B

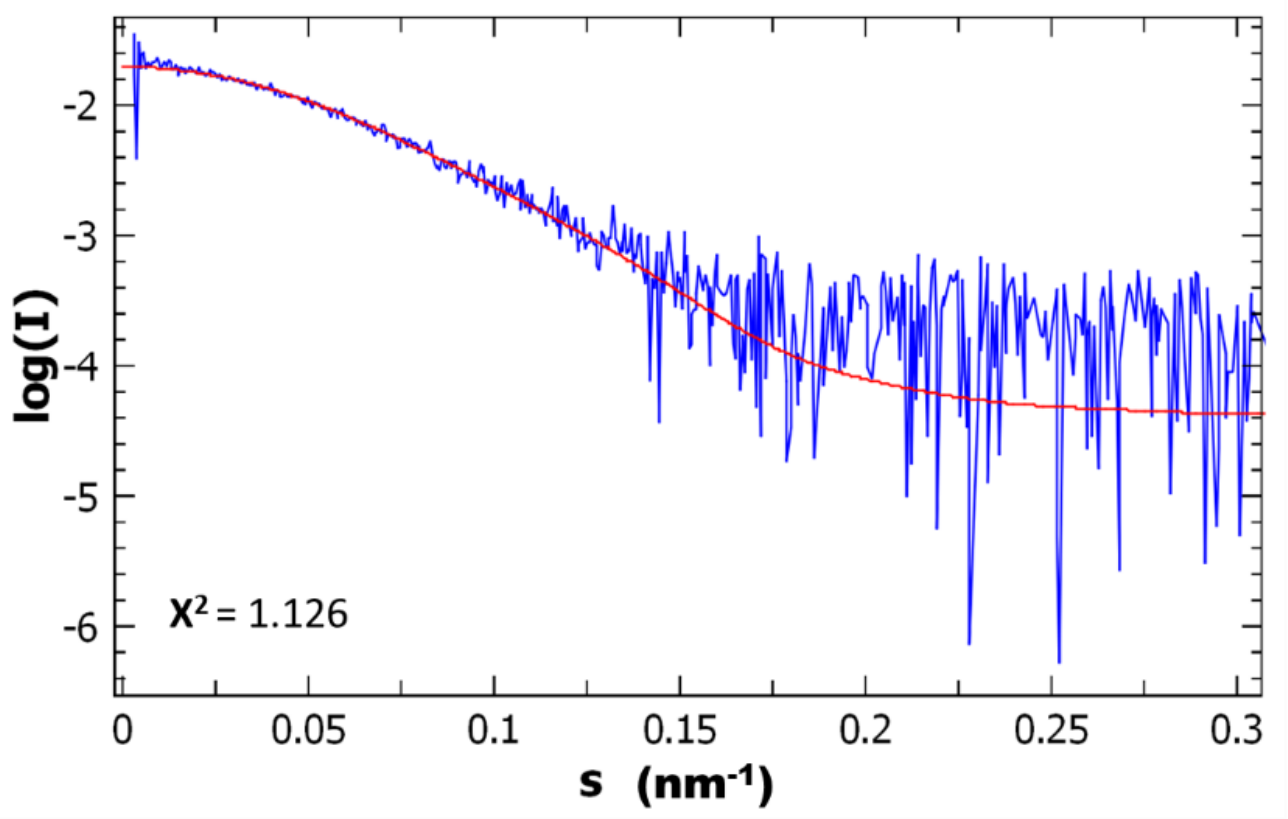

Figure 2. Size-exclusion chromatography coupled to small angle X-rays scattering (SEC-SAXS) analysis of CrRPI1. (A) High performance liquid chromatography (HPLC) chromatogram of CrRPI1 with absorption at $280 \mathrm{~nm}$ in red and at $260 \mathrm{~nm}$ in blue. (B) SAXS scattering curve $\log (\mathrm{I})=\mathrm{f}(\mathrm{s})$ in blue was fitted to the crystallographic dimeric model of CrRPI1 in red. 
A
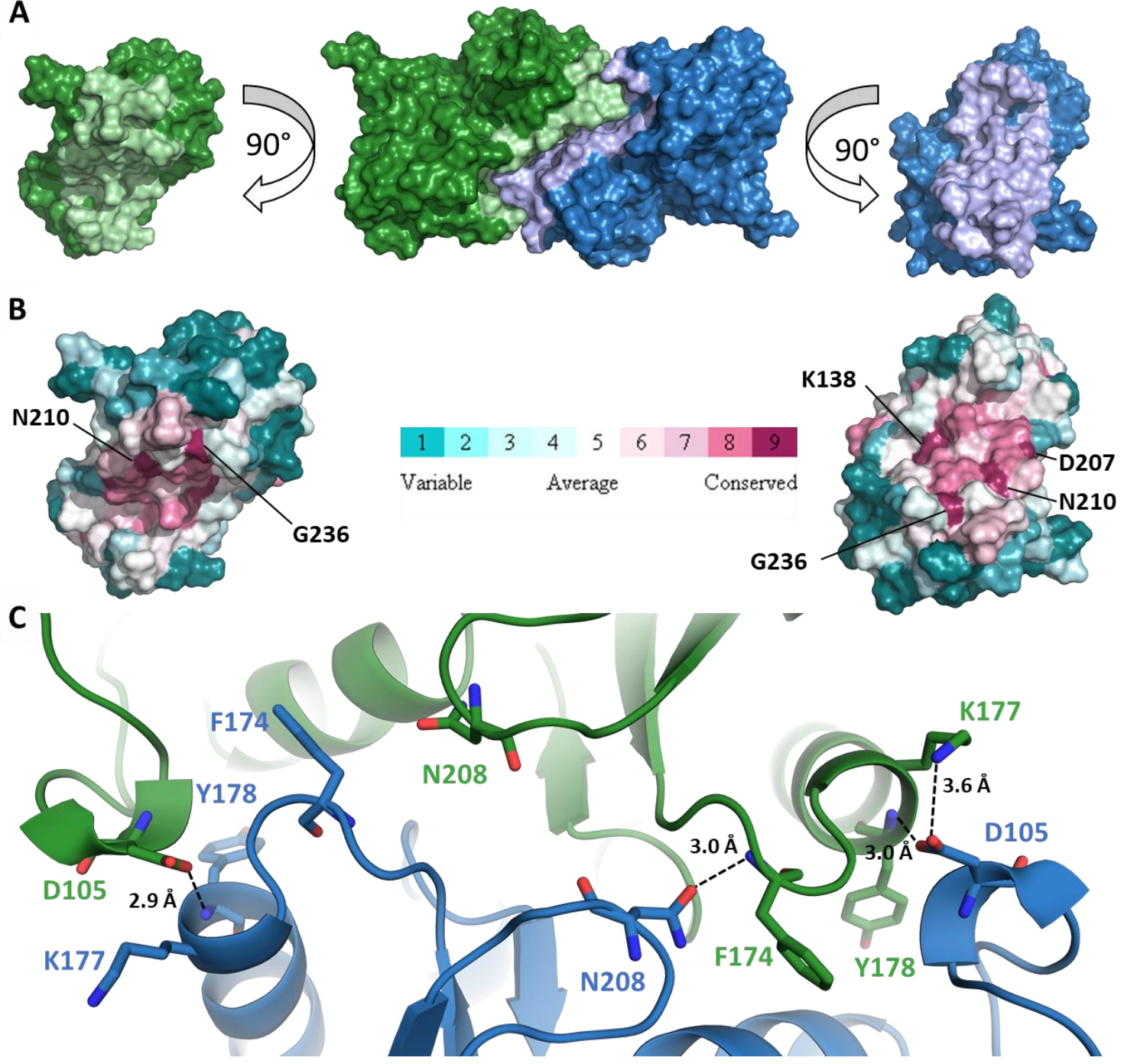

K177
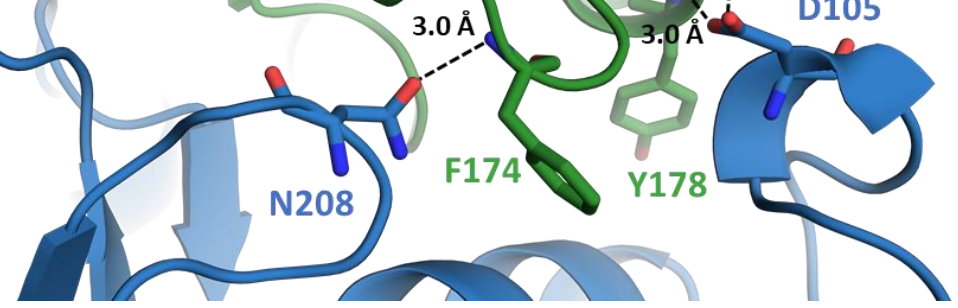

Figure 3. CrRPI1 homodimerization. (A) From left to right, Connolly solvent exclusion surface representation of subunit A, dimer and subunit B of CrRPI1. Subunit A is colored in green, and subunit $\mathrm{B}$ in blue. Residues composing the interface between the two subunits are colored in light green and light blue for subunits A and B respectively. (B) Surface residues conservation of subunits A and B as calculated with CONSURF colored from teal (least conserved) to purple (most conserved). Most conserved residues of the interface are annotated. (C) Cartoon representation of the dimer interface. Residues in bonding distances are represented in stick and annotated. Polar contacts are traced with black dashed lines with interatomic distances indicated in Ångström $(\AA ̊)$.

\subsection{RPI1 Catalytic Site}

CrRPI1 crystallographic model includes a sulfate ion, a sodium ion and water molecules located in a shallow pocket exposed to solvent (Figure 4A). Structural superposition of CrRPI1 with RpiA from Legionella pneumophila (LpRPI, PDB: 6MC0, RMSD $=0.734 \AA$ ), which is co-crystalized with ribose-5-phosphate bound to the active site, reveals extended similarities (Figure S2). Surface charges were calculated on Pymol using the APBS electrostatics plugin $[27,28]$. For both enzymes, the active site is an analog cavity with an electronegative charge at the center, surrounded by electropositive charges at the rims (Figure 5A,B). Electrostatic surface potentials are similarly distributed on both active sites, suggesting an identical electrostatic complementarity toward the substrate. Indeed, an electropositive patch just outside the cavity is ideally positioned to accommodate the phosphate moiety of R5P in LpRPI (Figure 5B) and was found to anchor the co-crystallized sulfate ion in CrRPI1 (Figure 5A). 
The sulfate ion in CrRPI1 hence occupies the location of the phosphate of the ribose-5-phosphate (Figure 5A,B) supporting a conserved mode of substrate recognition. This sulfate ion is coordinated by polar contacts with the side chains of S60, T61, and K155 and the main chain amine of T61. Residues K37, G59, S60, T61, A62, I150, and K155 principally contribute to the rest of the pocket surface in CrRPI1 (Figure 4B). All these residues are highly conserved in all species from the three kingdoms of life (Figure S1). Water molecules in CrRPI1 are placed where R5P carbon skeleton binds LpRPI. The sodium ion co-crystallized with CrRPI1 lies where the aldehyde function of R5P binds LpRPI. Residues D115 and E137 have been proposed to play a critical role in the catalytic mechanism [3,29]. In our model of CrRPI1, their side chains are positioned at, respectively, $3.1 \AA$ and $6.4 \AA$ away from the sodium ion, allowing their interaction with the carbonyl function of the substrate. Side chains rearrangements and water displacements are expected for the formation of the Michaelis complex and the subsequent catalytic isomerization.

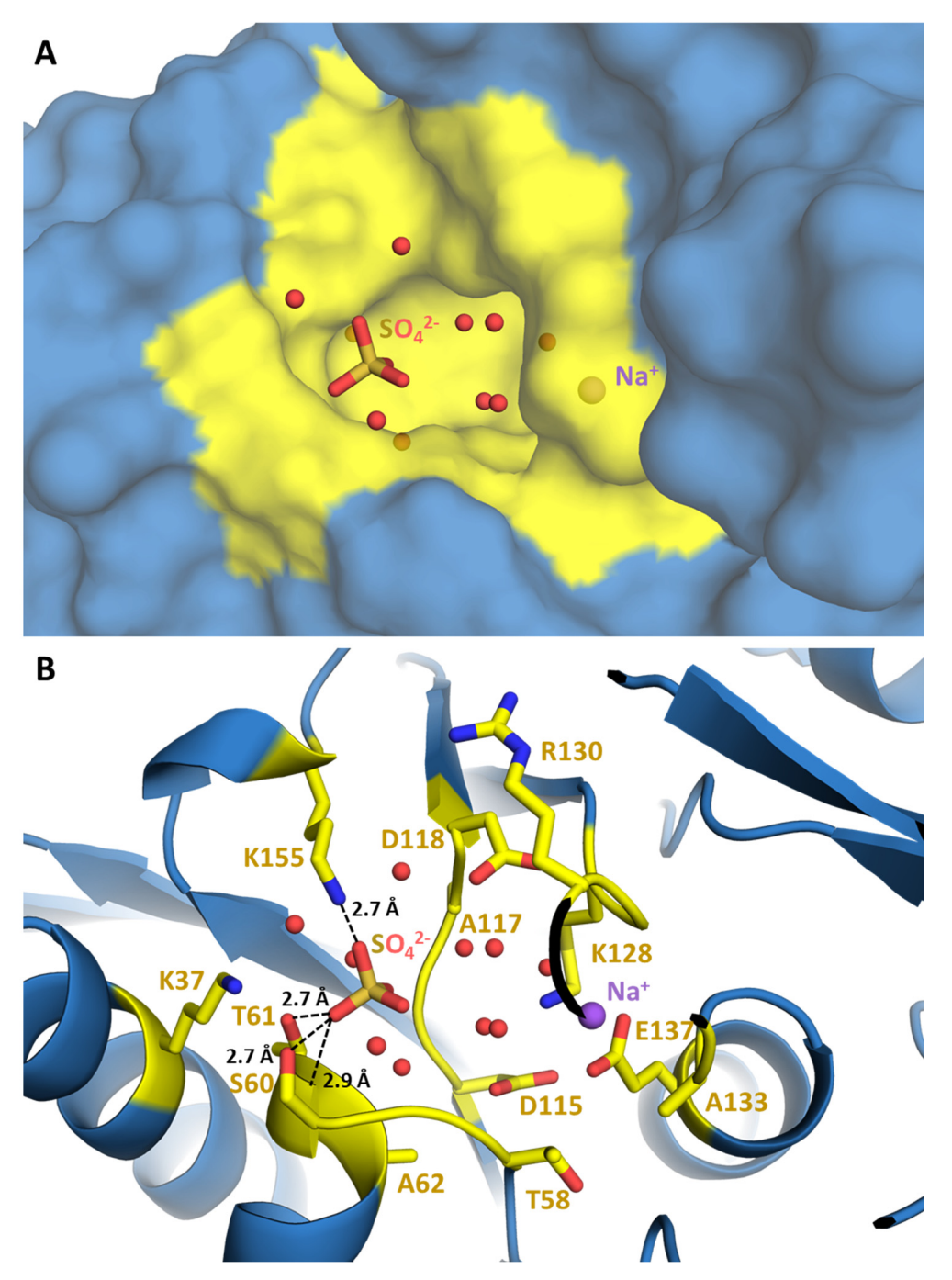

Figure 4. CrRPI1 catalytic pocket. (A) The Connolly solvent exclusion surface of the catalytic cleft of CrRPI1 with ligands in it (sulfate ion, water, sodium ion). Residues contributing to the cavity are colored in yellow, other residues are colored in blue. (B) The same view of the catalytic cleft as in (A). The protein is colored in blue and represented in cartoon. Residues contributing to the catalytic cleft are colored in yellow and their side chains are represented in sticks. Polar contacts between ligand and residues are represented in black dashed lines with corresponding interatomic distances indicated in Ångströms (Å). 


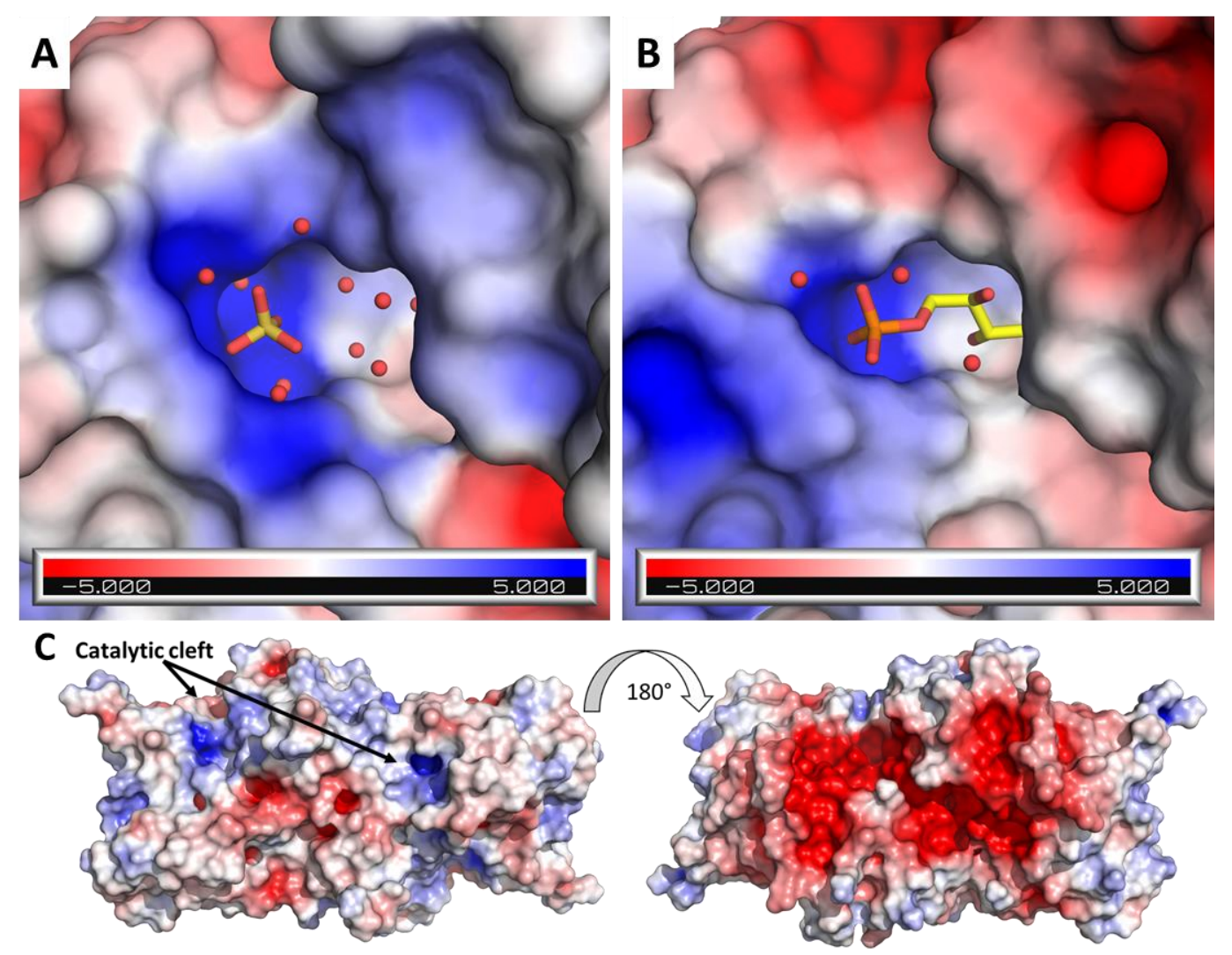

Figure 5. Electrostatic potential of RPI surface. (A) View of the catalytic cleft of CrRPI1 electrostatic surface calculated by PyMOL APBS and represented in a gradient from blue (electropositive) to red (electronegative). The sulfate ion is represented in sticks and water molecules as spheres. (B) View of the catalytic cleft of Legionella pneumophila RpiA of PDB model 6MC0, superposed to CrRPI1 model and colored as in A. Co-crystallized ribose-5-phosphate is represented in sticks. (C) Electronegative patch of CrRPI1 dimer. Electrostatic surface of CrRPI1 dimer facing the catalytic cleft up and with a $180^{\circ}$ rotation from this position was calculated by PyMOL APBS.

\subsection{CrRPI1 Sites of Post-Translational Modifications}

All CBC enzymes undergo diverse types of redox post-translational modifications including disulfide bond formation, glutathionylation or nitrosylation, under the control of thioredoxins (TRX) and glutaredoxins (GRX) [15,20]. CrRPI1 contains four cysteines: C149, C250 and C192 are conserved in green microalgae, while C175 is present in algae and land plants (Figure S1). Two cysteines (C149 and C175) were identified as reactive in vivo through a high-throughput screen, and C149 was further identified as a putative TRX target [20]. CrRPI1 was also identified as a target of S-nitrosylation or S-glutathionylation in proteomic studies [16,30]. In our CrRPI1 crystal structure, C149 and C250 position their sulfur atoms $4.4 \AA$ apart, a distance compatible with the formation of a disulfide bridge. C149 and C250 side chains are modelled as free thiols (Figure 1A and Figure S3). Both side chains are buried in the protein hydrophobic core where TRX or GRX should be excluded once RPI1 is folded. The two other cysteines of CrRPI1 are closer to the surface but separated by $20 \AA$, precluding the formation of an intramolecular disulfide bridge. C192 has a solvent exposed backbone and its sidechain points toward the hydrophobic core. C175 has its sulfur atom exposed to solvent and is the most likely to be the target of redox post translational modifications, such as the formation of an intermolecular disulfide bridge with another dimer of CrRPI1 or with a partner protein. Indeed, CrRPI1 is predicted to interact with ten other proteins in Chlamydomonas reinhardtii by the STRING database [31] and a disulfide bridge may stabilize or condition these interactions. Phosphoglycerate kinase 1 (PGK1), transketolase 1 (TRK1), ribulose-5-phosphate 3-epimerase 1 (RPE1) 
and 2 (RPE2), and phosphoribulokinase (PRK) are predicted to belong to this interaction network and are also part of the CBC and proven targets of redox regulation [20,32,33]. Protein interactions may hence contribute to the redox regulation of the $\mathrm{CBC}$ under varying physiological and environmental conditions. Furthermore TRK, RPI, RPE, and PRK activities are consecutive in the cycle, which may allow them to constitute an enzymatic metabolon.

A large electronegative patch is present at the back side of the dimer, $20 \AA$ away from the catalytic pocket (Figure 5 C). It spans both subunits and forms a continuous ellipsoid of approximately $55 \AA$ in length and $30 \AA$ in width. This extended surface patch is a candidate docking zone for additional partner or regulatory proteins. For instance, photosynthetic redox activator CrTRXf2 was reported to expose an electropositive crown of residues around its active site [34] and a large electropositive crescent surrounds CrPRK active site [35]. Such putative interactants or others bearing similar electropositive surfaces would favorably interact on the back side of CrRPI1 by electrostatic complementarity.

Phosphoproteomic studies [21] identified two phosphorylated amino acids on CrRPI1: T61 and S87 (Figure S3). Both are localized on the same surface as the catalytic cavity, T61 hydroxyl group being at $2.7 \AA$ of one of the oxygens of aforementioned active site sulfate. S87 is positioned $13.2 \AA$ away from T61, at the opposite side of the catalytic cleft at $7.1 \AA$ of E137. Phosphorylation of T61 would insert a bulky negatively charged group, which would probably preclude the binding of phosphate from the R5P substrate. Phosphorylation of T61 would thus outcompete the binding of R5P. S87 phosphorylation may play a less direct role in the regulation of R5P binding. Both T61 and S87 are fully conserved from bacteria to plants and metazoans (Figure S1).

\section{Discussion}

We describe the first structure of a ribose-5-phosphate isomerase from a Viridiplanta, the model microalga Chlamydomonas reinhardtii. The structural fold of CrRPI1 (Figure 1B) is similar to previously described structures of canonical $\mathrm{RPI}_{\mathrm{A}}$ [29] even though plastidial RPI participates in both the Calvin-Benson cycle and the pentose phosphate pathway. CrRPI1 catalytic site is described free from substrate and product, representing the apo state of the enzyme. Six water molecules, a sulfate ion and a sodium ion are however present and therefore allow to predict the coordination and placement of its substrate R5P in this pocket through structural superposition and sequence analysis. Most of the residues shaping the catalytic pocket are strictly conserved (Figure S1), supporting a conserved catalytic mechanism for all $\mathrm{RPI}_{\mathrm{A}}$ described to date [4].

CrRPI1 is a homodimer in solution and in the crystal packing (Figure 1A, Figure 2A,B, and Figure 3A-C). This homodimeric organization was previously observed (L. pneumophila PDB 6MC0). RPI was alternatively reported to form homotetramers in other species such as Saccharomyces cerevisiae or archaea [36,37]. The oligomerization appears to depend on the length of the 197-204 loop and the interaction network of the conserved residue R197, as suggested based on the S. cerevisiae structure [36]. In CrRPI 1 homodimer, the distance between catalytic residues $\mathrm{D} 115_{\mathrm{A}}$ and $\mathrm{D} 115_{\mathrm{B}}$ is $40 \AA$. Whether homodimerization induces cooperativity between the active sites will be tested in future studies.

By contributing to the Calvin-Benson cycle and to the pentose phosphate pathway [2,38], CrRPI1 activity should be subject to several, maybe mutually exclusive, regulations of its catalytic parameters. Structural analysis suggests several possible, mutually permissive regulation mechanisms. First, CrRPI1 was identified in C. reinhardtii thioredoxome [20], as a consequence of its affinity with the dithiol-disulfide exchanger thioredoxin. Hence, RPI1 is likely to be activated in concert with other CBC enzymes, such as PRK [35]. The presence of only cysteines 149 and 250 at disulfide bonding distance, but not accessible to solvent, argues in favor of irreversible redox states of the cysteine pair once the protein is folded. The two other cysteines 175 and 192 side chains are close to or exposed to solvent. They are modelled in CrRPI1 at a distance of $20 \AA$ which precludes the formation of an intramolecular disulfide bridge. Alternatively, other redox post translational modifications of these cysteines, such as intermolecular disulfide bridges, S-glutathionylation, S-nitrosylation may occur as suggested by 
proteomic studies $[16,20,30]$. Interestingly, RPI1 could also interact with partner proteins, including through intermolecular disulfide bridges. The formation of multienzymatic complexes with following and preceding enzymes from the $\mathrm{CBC}$ may allow channeling of substrates as suggested by a study on flux distribution and metabolic pools in Chlamydomonas reinhardtii chloroplast [39]. This hypothesis has to be tested by isolation of such complexes from cellular extracts.

Regulation of the isomerase activity could also be achieved by phosphorylation of residues T61 and S87. Addition of a phosphate group onto T61 hydroxyl would block the entrance of the catalytic pocket with a negatively charged steric hindrance at the R5P and Ru5P binding site. This hypothesis seems attractive as, most importantly, these two residues are highly conserved throughout all analyzed species. This might indicate a ubiquitous, ancestral regulation rather than a CBC-specific control. The identity of the kinase/phosphatase couple that specifically targets CrRPI1 will be addressed during future research.

In spite of its long evolution in the photosynthetic lineage, chloroplastic CrRPI1 seems to have no major structural difference with previously described RPI of the A-type. Some putative regulatory elements are diverging from other studied $\mathrm{RPI}_{\mathrm{A}}$ and will need further functional characterizations. The co-regulations exerted by multiples signaling pathways on RPI, the coordinated regulations on RPI, and the rest of the metabolic pathways it belongs to should be further computed in a systems biology perspective. For this purpose, our high-resolution crystal structure of the first chloroplastic ribose-phosphate isomerase serves as a general blueprint for the analytic and synthetic biochemistry of an essential step of photosynthesis.

\section{Materials and Methods}

\subsection{Cloning}

The amino acid sequence of Chlamydomonas reinhardtii ribose-5-phosphate isomerase (Cre03.g187450, UniRef100 entry A8IRQ1) was analyzed with TargetP2.0 [40], ChloroP [41] and Predalgo [8] to predict the transit peptide cleavage site. The subsequent mature sequence of chloroplastic protein coding for amino acids 23 to 269 was amplified by PCR using a forward primer introducing an NcoI restriction site (bolded) at the start codon: 5'-CGCACCATGGCCGCGCCGGTCTCAA-3' and a reverse primer introducing a BamHI restriction site (bolded) downstream of the stop codon: 5'-GCACGGGATCCTTAGTGCTTCTTGGGGTTGGG-3' as previously reported [30]. The PCR was performed using the Chlamydomonas reinhardtii EST index [42] database plasmid AV390085 obtained from the Kazusa DNA Research Institute (Chiba, Japan). After purification, the PCR product was digested by NcoI and BamHI and cloned into the NdeI and BamHI restriction sites of a modified pET-3d-His vector allowing expression of the protein with a polyhistidine-tag at the N-terminus. The resulting plasmid, pET3d-His-CrRPI1 was then validated by Sanger sequencing.

\subsection{Protein Expression and Purification}

Recombinant CrRPI1 was produced using the Escherichia coli strain BL21(DE3) Rosetta-2 pLysS (Novagen Merck, Darmstadt, Germany) transformed with the pET3d-His6-CrRPI1 plasmid. Bacteria were grown in LB medium supplemented with ampicillin $(100 \mu \mathrm{g} / \mathrm{mL})$ at $37^{\circ} \mathrm{C}$ until the culture reached exponential phase at an optical density at $600 \mathrm{~nm}$ of 0.4 . The expression of RPI was then induced by addition of $0.2 \mathrm{mmol} / \mathrm{L}$ of IPTG and incubation at $27^{\circ} \mathrm{C}$ for three hours. Cells were harvested by centrifugation $5000 \mathrm{rcf}$ for $10 \mathrm{~min}$ at $4{ }^{\circ} \mathrm{C}$, resuspended in $30 \mathrm{mmol} / \mathrm{L}$ Tris-HCL pH $=7.9$, $0.5 \mathrm{mmol} / \mathrm{L}$ EDTA (buffer A), and lysed by $2 \mathrm{~min}$ sonication with $0.4 \mathrm{sec}$ pulses at output 5 on W-375 sonicator equipped with microtip (Qsonica, Newtown, CT, USA). The total extract was then centrifuged at 20,000 rcf for $20 \mathrm{~min}$ and the soluble fraction loaded on an affinity chromatography with $2 \mathrm{~mL}$ of NiNTA resin (Sigma-Aldrich Merck, Darmstad, Germany). The resin was washed with Buffer A supplemented with increasing concentrations of imidazole $(10 \mathrm{mmol} / \mathrm{L}, 20 \mathrm{mmol} / \mathrm{L}$, and $30 \mathrm{mmol} / \mathrm{L}$ ). CrRPI1 was then eluted in with buffer A supplemented with $100 \mathrm{mmol} / \mathrm{L}, 200 \mathrm{mmol} / \mathrm{L}$ 
and $300 \mathrm{mmol} / \mathrm{L}$ of imidazole. Fractions were then analyzed by SDS-PAGE on a 12\% acrylamide gel revealed by Coomassie blue staining. Fractions containing purified CrRPI1 were pooled, and buffer exchanged against buffer A prior to being concentrated by ultrafiltration on 3000 MWCO filter units (Millipore Merck, Darmstadt, Germany). A final concentration of $9 \mathrm{mg} \cdot \mathrm{mL}^{-1}$ was measured by NanoDrop 2000 spectrophotometer (Thermo Fisher Scientific, Waltham, MA, USA) with theoretical $\mathrm{Mw}=27,551 \mathrm{~g} \cdot \mathrm{mol}^{-1}$ and $\varepsilon=14,565 \mathrm{~mol}^{-1} \cdot \mathrm{L} \cdot \mathrm{cm}^{-1}$. Proteins were then analyzed by injection on a Superose 6 increase size exclusion chromatography column in buffer C (20 mM Tris- $\mathrm{HCl} \mathrm{pH} 7.9$, $100 \mathrm{mmol} / \mathrm{L} \mathrm{NaCl}$ ).

\subsection{Protein Crystallization and Structure Determination}

Purified CrRPI1 was tested for crystallization on commercial sparse-screening conditions (Qiagen, Hilden Germany) of the Joint Center for Structural Genomics screens [43] and JBScreen classic 1-8 (Jena Bioscience, Jena, Germany) with a mixture of $50 \mathrm{~nL}$ of protein and $50 \mathrm{~nL}$ of precipitant solution equilibrated against $30 \mu \mathrm{L}$ of reservoir solution at $20^{\circ} \mathrm{C}$. Two monocrystal grew after one year in conditions JBScreen 6-C8 ( 3 mmol. $\mathrm{L}^{-1}$ ammonium sulfate) and JBScreen 6-D9 (3 mmol. $\mathrm{L}^{-1}$ ammonium sulfate, $20 \%$ glycerol). Crystals were flash frozen in liquid nitrogen for diffraction experiments at Proxima-1 beamline of SOLEIL synchrotron (Gif sur Yvette, France) [44]. A 99.63\% complete dataset at $1.40 \AA$ resolution was obtained from 3600 images over $360^{\circ}$ of phi rotation and indexed in the $\mathrm{P} 21_{1}$ space group, integrated, scaled and converted with XDSME [45]. Structure was phased by molecular replacement with PHENIX [46,47], PHASER-MR [48] using a search model obtained from the Robetta server [49]. Two CrRPI1 monomers were found in the asymmetric unit. Model was then refined by iterative cycle of manual building in WinCOOT [50,51], followed by refinement with PHENIX.REFINE [52] until completion of a structure passing MOLPROBITY [53] evaluation with 100\% residues in Ramachandran restrains, RMS(bond) $=0.011$, RMS(angles) $=1.18$ and final Rwork $=0.1594$, Rfree $=0.1774$ (Table 1). Structure representations were drawn with PYMOL (Schrodinger, New York, NY, USA).

\subsection{SEC-SAXS Analysis}

Here, $50 \mu \mathrm{L}$ of pure CrRPI1 at a concentration of $10 \mathrm{mg} \cdot \mathrm{mL}^{-1}$ were injected on BioSEC-3 300 size-exclusion chromatography column (Agilent Technologies, Santa Clara, CA, USA) equilibrated in buffer $\mathrm{C}$, in series with the small-angle X-rays scattering (SAXS) exposure capillary at the synchrotron beamline SWING (SOLEIL, Gif sur Yvette, France). Collected scattering images were analyzed on the application Foxtrot 3.3.4 (Xenocs, Sassenage, France) and the ATSAS 2.8.3 suite [54,55]. PrimusQT calculated a radius of gyration of $28.16 \pm 0.18 \AA$ and estimated SAXS CrRPI1 molecular weight at 52,781 Da (Qp), 53,991 Da (MoW), 49,221 (Vc), and 55,257 Da (size and shape). This molecular weight allows us to fit between 1.78 and 2.00 times the molecular weight of monomeric recombinant CrRPI1 estimated with Expasy ProtParam at 27,551 Da.

\subsection{Structural Data}

Crystallographic data are registered at the Protein Data Bank under accession code 6ZXT.

Supplementary Materials: Supplementary materials can be found at http://www.mdpi.com/1422-0067/21/20/ $7787 /$ s1. Figure S1. Sequence alignment made by Clustal Omega of RPI $\mathrm{A}_{\mathrm{A}}$ from different species. Residues with more than $50 \%$ conservation are colored in red, strictly conserved residues are in white with a red background. Numbering is according to Chlamydomonas reinhardtii. RPI sequences were retrieved from uniprotKB database for the following species: Chlamydomonas reinhardtii (A8IRQ1), Glycine Max (I1NC83), Arabidopsis thaliana (Q9S726), Plasmodium falciparum (Q8I3W2), Deinococcus radiodurans (Q9RW24), Streptococcus pneumoniae (B2INU5), Mus musculus (P47968), Homo sapiens (P49247), Gonium pectorale (A0A150H4N9), Volvox carteri f. nagariensis (D8TV46), Chlorella sorokiniana (A0A2P6TRF5), Raphidocelis subcapitata (A0A2V0PB57), Chlorella variabilis (E1Z7C4), Chlamydomonas eustigma (A0A250XAH6), Polytomella sp. Pringsheim 198.80 (K0J8U0), Thermus thermophilus (Q72J47), Synechocystis sp. PCC6803 (Q55766), Burkholderia thailandensis (A0A2Z4SMZ7), Legionella pneumophila (A5I9R2). Figure S2. Comparison of the catalytic clefts of CrRPI1 and LpRPI (PDB entry 6MC0). (A) Structures are aligned $(\mathrm{RMSD}=0.701 \AA)$ and represented in cartoon with side chains of catalytic pocket colored in magenta and 
represented as sticks. Other residues of CrRPI1 and LpRPI are respectively colored in blue and green. Sulfate ion and Ribose-5-phosphate are represented in sticks, water molecules and Na ion are represented in spheres. (B) Structure of CrRPI1. (C) Structure of LpRPI. Figure S3. (A) CrRPI1 post-translational modifications sites. Residues of the catalytic cleft are colored in orange. Cysteines and residues found phosphorylated in Wang, Gau et al. are represented in sticks and respectively colored in red and blue. (B) Connolly solvent exclusion surface of the same view as A. and same color code.

Author Contributions: Conceptualization, S.D.L. and J.H.; investigation, T.L.M. and J.H.; resources, S.D.L. and J.H.; writing-original draft preparation, T.L.M. and J.H.; writing-review and editing, T.L.M., P.C., S.D.L., and J.H.; supervision, J.H.; funding acquisition, S.D.L. and J.H. All authors have read and agreed to the published version of the manuscript.

Funding: This research was funded by CNRS, Sorbonne Université and Agence Nationale de la Recherche Grants LABEX DYNAMO 11-LABX-0011 and JCJC CALVINTERACT ANR-19-CE11-0009.

Acknowledgments: We thank the FR 550 Institut de Biologie Physico-Chimique for the access to the crystallization platform and to the data visualization wall. We acknowledge SOLEIL beamlines Proxima-1 and Proxima-2a for providing the setup of X-ray diffraction experiments and beamline SWING for SAXS experiments. We thank Laure Michelet, Fernanda Borrega, and Sandrine Hoang for help with the preparation and characterization of the recombinant protein.

Conflicts of Interest: The authors declare no conflict of interest. The funders had no role in the design of the study; in the collection, analyses, or interpretation of data; in the writing of the manuscript, or in the decision to publish the results.

\section{References}

1. Pfannschmidt, T.; Yang, C. The hidden function of photosynthesis: A sensing system for environmental conditions that regulates plant acclimation responses. Protoplasma 2012, 249 (Suppl 2), S125-S136. [CrossRef]

2. Benson, A.A.; Bassham, J.A.; Calvin, M.; Hall, A.G.; Hirsch, H.E.; Kawaguchi, S.; Lynch, V.; Tolbert, N.E. The path of carbon in photosynthesis. XV. Ribulose and sedoheptulose. J. Biol. Chem. 1952, 196, 703-716. [PubMed]

3. Zhang, R.; Andersson, C.E.; Savchenko, A.; Skarina, T.; Evdokimova, E.; Beasley, S.; Arrowsmith, C.H.; Edwards, A.M.; Joachimiak, A.; Mowbray, S.L. Structure of Escherichia coli ribose-5-phosphate isomerase: A ubiquitous enzyme of the pentose phosphate pathway and the Calvin cycle. Structure 2003, 11, 31-42. [CrossRef]

4. Chen, J.; Wu, H.; Zhang, W.; Mu, W. Ribose-5-phosphate isomerases: Characteristics, structural features, and applications. Appl. Microbiol. Biotechnol. 2020, 104, 6429-6441. [CrossRef] [PubMed]

5. Stincone, A.; Prigione, A.; Cramer, T.; Wamelink, M.M.; Campbell, K.; Cheung, E.; Olin-Sandoval, V.; Gruning, N.M.; Kruger, A.; Tauqeer Alam, M.; et al. The return of metabolism: Biochemistry and physiology of the pentose phosphate pathway. Biol. Rev. Camb. Philos. Soc. 2015, 90, 927-963. [CrossRef]

6. Sasso, S.; Stibor, H.; Mittag, M.; Grossman, A.R. From molecular manipulation of domesticated Chlamydomonas reinhardtii to survival in nature. eLife 2018, 7, e39233. [CrossRef] [PubMed]

7. Merchant, S.S.; Prochnik, S.E.; Vallon, O.; Harris, E.H.; Karpowicz, S.J.; Witman, G.B.; Terry, A.; Salamov, A.; Fritz-Laylin, L.K.; Marechal-Drouard, L.; et al. The Chlamydomonas genome reveals the evolution of key animal and plant functions. Science 2007, 318, 245-250. [CrossRef] [PubMed]

8. Tardif, M.; Atteia, A.; Specht, M.; Cogne, G.; Rolland, N.; Brugiere, S.; Hippler, M.; Ferro, M.; Bruley, C.; Peltier, G.; et al. PredAlgo: A new subcellular localization prediction tool dedicated to green algae. Mol. Biol. Evol. 2012, 29, 3625-3639. [CrossRef] [PubMed]

9. Johnson, X.; Alric, J. Central carbon metabolism and electron transport in Chlamydomonas reinhardtii: Metabolic constraints for carbon partitioning between oil and starch. Eukaryot. Cell 2013, 12, 776-793. [CrossRef]

10. Klein, U. Compartmentation of glycolysis and of the oxidative pentose-phosphate pathway in Chlamydomonas reinhardii. Planta 1986, 167, 81-86. [CrossRef]

11. Strenkert, D.; Schmollinger, S.; Gallaher, S.D.; Salome, P.A.; Purvine, S.O.; Nicora, C.D.; Mettler-Altmann, T.; Soubeyrand, E.; Weber, A.P.M.; Lipton, M.S.; et al. Multiomics resolution of molecular events during a day in the life of Chlamydomonas. Proc. Natl. Acad. Sci. USA 2019, 116, 2374-2383. [CrossRef] [PubMed] 
12. Hammel, A.; Sommer, F.; Zimmer, D.; Stitt, M.; Mühlhaus, T.; Schroda, M. Overexpression of sedoheptulose-1,7-bisphosphatase enhances photosynthesis in Chlamydomonas reinhardtii and has no effect on the abundance of other Calvin-Benson cycle enzymes. bioRxiv 2020. [CrossRef]

13. Mettler, T.; Muhlhaus, T.; Hemme, D.; Schottler, M.A.; Rupprecht, J.; Idoine, A.; Veyel, D.; Pal, S.K.; Yaneva-Roder, L.; Winck, F.V.; et al. Systems Analysis of the Response of Photosynthesis, Metabolism, and Growth to an Increase in Irradiance in the Photosynthetic Model Organism Chlamydomonas reinhardtii. Plant Cell 2014, 26, 2310-2350. [CrossRef] [PubMed]

14. Scheibe, R. Redox-modulation of chloroplast enzymes: A common principle for individual control. Plant Physiol. 1991, 96, 1-3. [CrossRef] [PubMed]

15. Michelet, L.; Zaffagnini, M.; Morisse, S.; Sparla, F.; Perez-Perez, M.E.; Francia, F.; Danon, A.; Marchand, C.H.; Fermani, S.; Trost, P.; et al. Redox regulation of the Calvin-Benson cycle: Something old, something new. Front. Plant Sci. 2013, 4, 470. [CrossRef]

16. Morisse, S.; Zaffagnini, M.; Gao, X.H.; Lemaire, S.D.; Marchand, C.H. Insight into protein S-nitrosylation in Chlamydomonas reinhardtii. Antioxid. Redox. Signal. 2014, 21, 1271-1284. [CrossRef]

17. Michelet, L.; Zaffagnini, M.; Vanacker, H.; Le Marechal, P.; Marchand, C.; Schroda, M.; Lemaire, S.D.; Decottignies, P. In vivo targets of S-thiolation in Chlamydomonas reinhardtii. J. Biol. Chem. 2008, 283, 21571-21578. [CrossRef]

18. Huppe, H.C.; Buchanan, B.B. Activation of a chloroplast type of fructose bisphosphatase from Chlamydomonas reinhardtii by light-mediated agents. Z. Naturforsch. C J. Biosci. 1989, 44, 487-494. [CrossRef]

19. Pasquini, M.; Fermani, S.; Tedesco, D.; Sciabolini, C.; Crozet, P.; Naldi, M.; Henri, J.; Vothknecht, U.; Bertucci, C.; Lemaire, S.D.; et al. Structural basis for the magnesium-dependent activation of transketolase from Chlamydomonas reinhardtii. Biochim. Biophys. Acta Gen. Subj. 2017, 1861, 2132-2145. [CrossRef]

20. Perez-Perez, M.E.; Mauries, A.; Maes, A.; Tourasse, N.J.; Hamon, M.; Lemaire, S.D.; Marchand, C.H. The Deep Thioredoxome in Chlamydomonas reinhardtii: New Insights into Redox Regulation. Mol. Plant 2017, 10, 1107-1125. [CrossRef]

21. Wang, H.; Gau, B.; Slade, W.O.; Juergens, M.; Li, P.; Hicks, L.M. The global phosphoproteome of Chlamydomonas reinhardtii reveals complex organellar phosphorylation in the flagella and thylakoid membrane. Mol. Cell. Proteom. 2014, 13, 2337-2353. [CrossRef] [PubMed]

22. Holmes, M.A.; Buckner, F.S.; Van Voorhis, W.C.; Verlinde, C.L.; Mehlin, C.; Boni, E.; DeTitta, G.; Luft, J.; Lauricella, A.; Anderson, L.; et al. Structure of ribose 5-phosphate isomerase from Plasmodium falciparum. Acta Crystallogr. Sect. F Struct. Biol. Cryst. Commun. 2006, 62, 427-431. [CrossRef] [PubMed]

23. Essenberg, M.K.; Cooper, R.A. Two ribose-5-phosphate isomerases from Escherichia coli K12: Partial characterisation of the enzymes and consideration of their possible physiological roles. Eur. J. Biochem. 1975, 55, 323-332. [CrossRef] [PubMed]

24. Rutner, A.C. Spinach 5-phosphoribose isomerase. Purification and properties of the enzyme. Biochemistry 1970, 9, 178-184. [CrossRef] [PubMed]

25. Krissinel, E.; Henrick, K. Inference of macromolecular assemblies from crystalline state. J. Mol. Biol. 2007, 372, 774-797. [CrossRef]

26. Landau, M.; Mayrose, I.; Rosenberg, Y.; Glaser, F.; Martz, E.; Pupko, T.; Ben-Tal, N. ConSurf 2005: The projection of evolutionary conservation scores of residues on protein structures. Nucleic Acids Res. 2005, 33, W299-W302. [CrossRef]

27. Jurrus, E.; Engel, D.; Star, K.; Monson, K.; Brandi, J.; Felberg, L.E.; Brookes, D.H.; Wilson, L.; Chen, J.; Liles, K.; et al. Improvements to the APBS biomolecular solvation software suite. Protein Sci. Publ. Protein Soc. 2018, 27, 112-128. [CrossRef]

28. Dolinsky, T.J.; Czodrowski, P.; Li, H.; Nielsen, J.E.; Jensen, J.H.; Klebe, G.; Baker, N.A. PDB2PQR: Expanding and upgrading automated preparation of biomolecular structures for molecular simulations. Nucleic Acids Res. 2007, 35, W522-W525. [CrossRef]

29. Hamada, K.; Ago, H.; Sugahara, M.; Nodake, Y.; Kuramitsu, S.; Miyano, M. Oxyanion hole-stabilized stereospecific isomerization in ribose-5-phosphate isomerase (Rpi). J. Biol. Chem. 2003, 278, 49183-49190. [CrossRef] 
30. Zaffagnini, M.; Bedhomme, M.; Groni, H.; Marchand, C.H.; Puppo, C.; Gontero, B.; Cassier-Chauvat, C.; Decottignies, P.; Lemaire, S.D. Glutathionylation in the photosynthetic model organism Chlamydomonas reinhardtii: A proteomic survey. Mol. Cell. Proteom. 2012, 11, M111.014142. [CrossRef]

31. Szklarczyk, D.; Gable, A.L.; Lyon, D.; Junge, A.; Wyder, S.; Huerta-Cepas, J.; Simonovic, M.; Doncheva, N.T.; Morris, J.H.; Bork, P.; et al. STRING v11: Protein-protein association networks with increased coverage, supporting functional discovery in genome-wide experimental datasets. Nucleic Acids Res. 2019, 47, D607-D613. [CrossRef]

32. Morisse, S.; Michelet, L.; Bedhomme, M.; Marchand, C.H.; Calvaresi, M.; Trost, P.; Fermani, S.; Zaffagnini, M.; Lemaire, S.D. Thioredoxin-dependent redox regulation of chloroplastic phosphoglycerate kinase from Chlamydomonas reinhardtii. J. Biol. Chem. 2014, 289, 30012-30024. [CrossRef] [PubMed]

33. Marri, L.; Zaffagnini, M.; Collin, V.; Issakidis-Bourguet, E.; Lemaire, S.D.; Pupillo, P.; Sparla, F.; Miginiac-Maslow, M.; Trost, P. Prompt and easy activation by specific thioredoxins of calvin cycle enzymes of Arabidopsis thaliana associated in the GAPDH/CP12/PRK supramolecular complex. Mol. Plant 2009, 2, 259-269. [CrossRef] [PubMed]

34. Lemaire, S.D.; Tedesco, D.; Crozet, P.; Michelet, L.; Fermani, S.; Zaffagnini, M.; Henri, J. Crystal Structure of Chloroplastic Thioredoxin $\mathrm{f} 2$ from Chlamydomonas reinhardtii Reveals Distinct Surface Properties. Antioxidants 2018, 7, 171. [CrossRef] [PubMed]

35. Gurrieri, L.; Del Giudice, A.; Demitri, N.; Falini, G.; Pavel, N.V.; Zaffagnini, M.; Polentarutti, M.; Crozet, P.; Marchand, C.H.; Henri, J.; et al. Arabidopsis and Chlamydomonas phosphoribulokinase crystal structures complete the redox structural proteome of the Calvin-Benson cycle. Proc. Natl. Acad. Sci. USA 2019, 116, 8048-8053. [CrossRef]

36. Graille, M.; Meyer, P.; Leulliot, N.; Sorel, I.; Janin, J.; Van Tilbeurgh, H.; Quevillon-Cheruel, S. Crystal structure of the S. cerevisiae D-ribose-5-phosphate isomerase: Comparison with the archaeal and bacterial enzymes. Biochimie 2005, 87, 763-769. [CrossRef]

37. Ishikawa, K.; Matsui, I.; Payan, F.; Cambillau, C.; Ishida, H.; Kawarabayasi, Y.; Kikuchi, H.; Roussel, A. A hyperthermostable D-ribose-5-phosphate isomerase from Pyrococcus horikoshii characterization and three-dimensional structure. Structure 2002, 10, 877-886. [CrossRef]

38. Dickens, F. Recent advances in knowledge of the hexose monophosphate shunt. Ann. N. Y. Acad. Sci. 1958, 75, 71-94. [CrossRef]

39. Kuken, A.; Sommer, F.; Yaneva-Roder, L.; Mackinder, L.C.; Hohne, M.; Geimer, S.; Jonikas, M.C.; Schroda, M.; Stitt, M.; Nikoloski, Z.; et al. Effects of microcompartmentation on flux distribution and metabolic pools in Chlamydomonas reinhardtii chloroplasts. eLife 2018, 7, e37960. [CrossRef]

40. Emanuelsson, O.; Brunak, S.; von Heijne, G.; Nielsen, H. Locating proteins in the cell using TargetP, SignalP and related tools. Nat. Protoc. 2007, 2, 953-971. [CrossRef]

41. Emanuelsson, O.; Nielsen, H.; von Heijne, G. ChloroP, a neural network-based method for predicting chloroplast transit peptides and their cleavage sites. Protein Sci. Publ. Protein Soc. 1999, 8, 978-984. [CrossRef]

42. Asamizu, E.; Nakamura, Y.; Miura, K.; Fukuzawa, H.; Fujiwara, S.; Hirono, M.; Iwamoto, K.; Matsuda, Y.; Minagawa, J.; Shimogawara, K.; et al. Establishment of publicly available cDNA material and information resource of Chlamydomonas reinhardtii (Chlorophyta) to facilitate gene function analysis. Phycologia 2004, 43, 722-726. [CrossRef]

43. Lesley, S.A.; Wilson, I.A. Protein production and crystallization at the joint center for structural genomics. J. Struct. Funct. Genom. 2005, 6, 71-79. [CrossRef] [PubMed]

44. Coati, A.; Chavas, L.M.G.; Fontaine, P.; Foos, N.; Guimaraes, B.; Gourhant, P.; Legrand, P.; Itie, J.P.; Fertey, P.; Shepard, W.; et al. Status of the crystallography beamlines at synchrotron SOLEIL. Eur. Phys. J. Plus 2017, 132, 174. [CrossRef]

45. Legrand, P. XDSME: XDS Made Easier. 2017. Available online: https://github.com/legrandp/xdsme (accessed on 20 October 2020).

46. Adams, P.D.; Afonine, P.V.; Bunkoczi, G.; Chen, V.B.; Davis, I.W.; Echols, N.; Headd, J.J.; Hung, L.W.; Kapral, G.J.; Grosse-Kunstleve, R.W.; et al. PHENIX: A comprehensive Python-based system for macromolecular structure solution. Acta Crystallogr. D Biol. Crystallogr. 2010, 66, 213-221. [CrossRef] [PubMed] 
47. Adams, P.D.; Afonine, P.V.; Bunkoczi, G.; Chen, V.B.; Echols, N.; Headd, J.J.; Hung, L.W.; Jain, S.; Kapral, G.J.; Grosse Kunstleve, R.W.; et al. The Phenix software for automated determination of macromolecular structures. Methods 2011, 55, 94-106. [CrossRef] [PubMed]

48. McCoy, A.J.; Grosse-Kunstleve, R.W.; Adams, P.D.; Winn, M.D.; Storoni, L.C.; Read, R.J. Phaser crystallographic software. J. Appl. Crystallogr. 2007, 40, 658-674. [CrossRef] [PubMed]

49. Raman, S.; Vernon, R.; Thompson, J.; Tyka, M.; Sadreyev, R.; Pei, J.; Kim, D.; Kellogg, E.; DiMaio, F.; Lange, O.; et al. Structure prediction for CASP8 with all-atom refinement using Rosetta. Proteins 2009, 77 (Suppl. 9), 89-99. [CrossRef]

50. Emsley, P.; Cowtan, K. Coot: Model-building tools for molecular graphics. Acta Crystallogr. D Biol. Crystallogr. 2004, 60, 2126-2132. [CrossRef]

51. Emsley, P.; Lohkamp, B.; Scott, W.G.; Cowtan, K. Features and development of Coot. Acta Crystallogr. Sect. D Biol. Crystallogr. 2010, 66, 486-501. [CrossRef]

52. Afonine, P.V.; Grosse-Kunstleve, R.W.; Echols, N.; Headd, J.J.; Moriarty, N.W.; Mustyakimov, M.; Terwilliger, T.C.; Urzhumtsev, A.; Zwart, P.H.; Adams, P.D. Towards automated crystallographic structure refinement with phenix.refine. Acta Crystallogr. Sect. D Biol. Crystallogr. 2012, 68, 352-367. [CrossRef]

53. Chen, V.B.; Arendall, W.B., 3rd; Headd, J.J.; Keedy, D.A.; Immormino, R.M.; Kapral, G.J.; Murray, L.W.; Richardson, J.S.; Richardson, D.C. MolProbity: All-atom structure validation for macromolecular crystallography. Acta Crystallogr. D Biol. Crystallogr. 2010, 66, 12-21. [CrossRef] [PubMed]

54. Petoukhov, M.V.; Franke, D.; Shkumatov, A.V.; Tria, G.; Kikhney, A.G.; Gajda, M.; Gorba, C.; Mertens, H.D.; Konarev, P.V.; Svergun, D.I. New developments in the ATSAS program package for small-angle scattering data analysis. J. Appl. Crystallogr. 2012, 45, 342-350. [CrossRef] [PubMed]

55. Franke, D.; Petoukhov, M.V.; Konarev, P.V.; Panjkovich, A.; Tuukkanen, A.; Mertens, H.D.T.; Kikhney, A.G.; Hajizadeh, N.R.; Franklin, J.M.; Jeffries, C.M.; et al. ATSAS 2.8: A comprehensive data analysis suite for small-angle scattering from macromolecular solutions. J. Appl. Crystallogr. 2017, 50, 1212-1225. [CrossRef]

Publisher's Note: MDPI stays neutral with regard to jurisdictional claims in published maps and institutional affiliations.

(C) 2020 by the authors. Licensee MDPI, Basel, Switzerland. This article is an open access article distributed under the terms and conditions of the Creative Commons Attribution (CC BY) license (http://creativecommons.org/licenses/by/4.0/). 